\title{
Post-transcriptional and translational regulation of mRNA-like long non-coding RNAs by microRNAs in early developmental stages of zebrafish embryos
}

\author{
Kyung-Tae Lee ${ }^{1} \mathcal{E}$ Jin-Wu Nam ${ }^{1,2,3, *}$ \\ ${ }^{1}$ Department of Life Science, College of Natural Sciences, Hanyang University, ${ }^{2}$ Research Institute for Natural Sciences, Hanyang \\ University, ${ }^{3}$ Research Institute for Convergence of Basic Sciences, Hanyang University, Seoul 133791, Korea
}

\begin{abstract}
At the post-transcriptional and translational levels, microRNA (miRNA) represses protein-coding genes via seed pairing to the 3' untranslated regions (UTRs) of mRNA. Although working models of miRNA-mediated gene silencing are successfully established using miRNA transfections and knockouts, the regulatory interaction between miRNA and long non-coding RNA (IncRNA) remain unknown. In particular, how the mRNA-resembling IncRNAs with $5^{\prime}$ cap, $3^{\prime}$ poly(A)-tail, or coding features, are regulated by miRNA is yet to be examined. We therefore investigated the functional interaction between miRNAs and IncRNAs with/without those features, in miRNAtransfected early zebrafish embryos. We observed that the greatest determinants of the miRNA-mediated silencing of IncRNAs were the $5^{\prime}$ cap and $3^{\prime}$ poly(A)-tails in IncRNAs, at both the post-transcriptional and translational levels. The IncRNAs confirmed to contain $5^{\prime}$ cap, $3^{\prime}$ poly(A)-tail, and the canonical miRNA target sites, were observed to be repressed in the level of both RNA and ribosome-protected fragment, while those with the miRNA target sites and without 5' cap and $3^{\prime}$ poly(A)-tail, were not robustly repressed by miRNA introduction, thus suggesting a role as a miRNA-decoy. [BMB Reports 2017; 50(4): 226-231]
\end{abstract}

\section{INTRODUCTION}

MicroRNA (miRNA), one of the conserved class of small non-coding RNAs of $\sim 22$ nucleotides (nt), participates in the control of protein-coding gene expression via base pairing to the $3^{\prime}$ untranslated region (3' UTR) of messenger RNA (mRNA) (1). Since the discovery of their role in miRNA-mediated gene

${ }^{*}$ Corresponding author. Tel: +82-2-2220-2428; Fax: +82-2-22980319; E-mail: jwnam@hanyang.ac.kr

https://doi.org/10.5483/BMBRep.2017.50.4.025

Received 11 February 2017, Revised 12 February 2017, Accepted 21 March 2017

Keywords: IncRNA, miRNA, sORF, 3' poly(A)-tail, 5' cap silencing (MGS), the regulatory modes and mechanisms of action involved have been studied, comparing between miRNA- and mock-transfected cells, or wild type and miRNAknockout cells (2-6). The major determinants of efficient miRNA targeting include the conserved Watson-Crick base pairing (called seed pairing) between 3' UTR of mRNA and the miRNA seed region; additional base pairing include position 8 of miRNA (7mer-m8, $7 \mathrm{~m} 8$ site) and the presence of adenine opposing position 1 of miRNA (7mer-A1, 7A1 site), or both the additional base pairing at position 8 and the presence of adenine opposing position 1 of miRNA (8mer site) (1). With seed pairing, the global downregulation of mRNA targeted by miRNA was first evidenced by reverse transcription polymerase chain reaction (RT-PCR) experiments (7) and microarray data analysis (8); although, the first miRNA to be discovered (lin-4) was previously shown to control the expression of its target protein, LIN-14, at the translational level (9).

Multiple studies using miRNA-transfected cell lines (10-12), miRNA knockouts in zebrafish embryos (13) and mouse neutrophils (10, 11, 13), high-throughput RNA sequencing (RNA-seq), and ribosome-protected fragment (RPF) sequencing (Ribo-seq), have investigated the miRNA regulatory mechanisms, revealing that both post-transcriptional and translational regulation modes are involved in MGS. Debates over the relative contribution and order of the two regulatory modes have elucidated the dynamics of miRNA-mediated repression. In addition, miRNA-transfection in human cell lines and miRNA knockout experiments in mice revealed that the destabilization of target mRNAs, rather than translational repression, is most responsible for MGS $(10,11)$. Recent experiments using either a zygotic dicer mutant with significantly reduced levels of miR-430 (13) or miRNAtransfection (14), were conducted to study the early developmental stages of zebrafish embryos. These researches claim that the targets are translationally repressed early on $(\sim 4$ hours after miRNA transfection), and post-transcriptionally downregulated later ( $\sim 6$ hours after miRNA transfection) (13), thus describing an early translational repression and a later dominant destabilization of the target mRNAs.

Although the majority of MGS-related studies mainly deal

ISSN: 1976-670X (electronic edition)

Copyright (C) 2017 by the The Korean Society for Biochemistry and Molecular Biology

(c) This is an open-access article distributed under the terms of the Creative Commons Attribution Non-Commercial License (http://creativecommons.org/licenses/by-nc/4.0) which permits unrestricted non-commercial use, distribution, and reproduction in any medium, provided the original work is properly cited. 
with the protein-coding genes, a handful of studies have examined the interaction between miRNA and long noncoding RNA (IncRNA), which is the other class of non-coding RNA longer than $200 \mathrm{nt}$ (15-18). IncRNAs are k versatile, heterogeneous RNA molecules, involved in diverse biological processes, such as transcriptional, post-transcriptional, and translational regulation of gene expression (19). Although the concept of competing endogenous RNA (ceRNA) is not widely accepted, recent reports indicate the functional role of IncRNAs containing miRNA target sites as miRNA-decoys that quench the endogenous miRNAs to their binding sites (20, 21). Interestingly, some IncRNAs that quench the miRNAs via these sites get destabilized (22-24), whereas others are resistant to or not affected by the miRNA-mediated repression $(25,26)$. For example, a well-studied IncRNA, the metastasis associated lung adenocarcinoma transcript 1 (non-protein coding) (MALAT1), is known to be repressed by miR-9 (22). Similarly, PTENP1, a pseudogene transcript of the tumor suppressor phosphatase and tensin homolog (PTEN), is repressed by endogenous miRNA targeting, and quenches the endogenous miRNAs (e.g., miR-20a, miR-19b, and miR-21), thus inhibiting the targeting of other mRNAs (23). Conversely, although the circular IncRNA (ciRS-7) that binds to miRNA-7 thorough its canonical target sites quenches miRNA, it is not repressed by MGS (25). So far, the factors that determine miRNA regulatory modes remain unknown.

IncRNAs lacking the open reading frames (ORFs) and protein-coding potential, share similar characteristics with protein-coding genes in terms of post-transcriptional processing (27). A substantial number, but not all of the IncRNAs, undergo similar post-transcriptional processing, such as $5^{\prime}$ capping, 3' polyadenylation, and splicing (28). However, recent studies using Ribo-seq and mass spectrometry revealed that IncRNAs containing small ORFs (sORFs) translate small peptides (29-33). Moreover, 4 mouse and 12 human IncRNAs are now known to have sORFs, as predicted by Ribo-seq. Considering that IncRNAs containing $5^{\prime}$ cap, 3' poly(A)-tail, and sORFs resemble protein-coding mRNAs in terms of having a similar structure, the IncRNAs with these three features may have regulatory functions (i.e., post-transcriptional and translational gene silencing) that are similar to those of protein-coding genes.

\section{RESULTS}

\section{High-confidence set of IncRNAs}

To acquire a high-confidence set of IncRNAs, the public IncRNA gene annotations (34-36) were re-examined, and a high-confidence set of mRNA-like IncRNAs embedding a 5' cap, 3' poly(A), sORF, or all three, were built (Supplementary Fig. S1). We first checked whether the IncRNAs are fragmented by examining the exon junctions between the IncRNAs and neighboring genes using publicly available RNA-seq data from the three early developmental stages in zebrafish (see Supplementary Material for more details). Both ends of the full-length IncRNAs were then updated with transcription start sites (TSSs) and cleavage and polyadenylation sites (CPSs), which denote the presence of $5^{\prime}$ cap and 3' poly(A)-tail respectively; these were predicted from cap analysis gene expression sequencing (CAGE-seq) (37) and poly(A)-position profiling by sequencing (3P-seq) (38) data from the same developmental stages, respectively (Fig. 1A; see Supplementary Material for more details). If CPS was distant from the $3^{\prime}$ end of the transcript, only CPS supported by the newly assembled transcript was assigned to the corresponding transcript. This led to the identification of 4,276 IncRNAs that contain CPS, 3,598 that contain TSS, and 1,959 that contain both TSS and CPS (Fig. 1B). Next, the coding potential of IncRNAs was examined by searching for sORFs with $3 \mathrm{nt}$ periodicity (Supplementary Fig. S2; see Supplementary Material for more details) in the publicly available Ribo-seq data from stage-matched samples, using Ribotaper (30). Consequently, 812 IncRNAs were predicted to be mRNA-like IncRNAs that have a putative sORF encoding less than 100 amino acids (Fig. 1B). In addition, 512 IncRNAs were predicted to have a putative ORF equal to or greater than 100 amino acids, and were analyzed separately from the IncRNAs discovered to have sORFs. Among the 8,534 IncRNAs predicted to have no ORFs, 1,083 were predicted as protein-coding mRNAs using the Coding Potential Calculator (CPC) (39), and were excluded from the IncRNA set without ORFs, thus yielding 7,451 IncRNAs without any ORF (Fig. 1B). For protein-coding mRNAs, the CPS and TSS updates were applied for better profiling of expression levels, as was done for IncRNAs. In

A

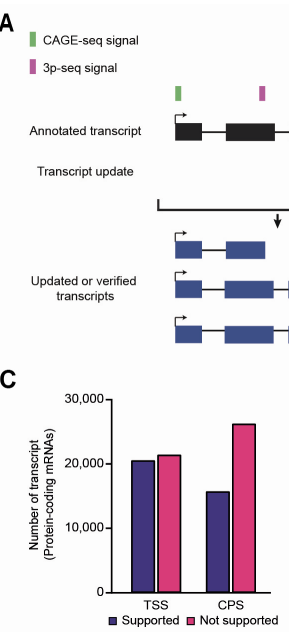

B
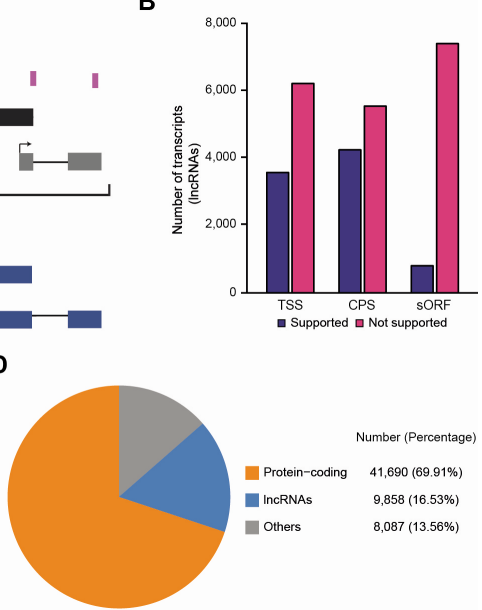

Fig. 1. mRNA-like IncRNA annotations. (A) A schematic flow of TSS and CPS updates of transcripts. (B) Numbers of IncRNAs with TSSs, CPSs, or sORFs. (C) Numbers of protein-coding mRNAs with TSSs or CPSs. (D) Composition of zebrafish transcripts after the TSS and CPS updates. 
total, 20,403 mRNAs contained TSS and 15,576 contained CPS (Fig. 1C), showing a higher proportion than that found for IncRNAs, which is consistent with observations in a previous study (40). For all the transcript annotations, $\sim 70 \%(41,690)$ were protein-coding transcripts, $\sim 17 \%(9,858)$ were IncRNA transcripts, and the remainder was considered as other ncRNA transcripts (Fig. 1D).

The stability of IncRNAs is dependent on mRNA-like features Since protein-coding mRNAs are more abundant and stable than IncRNA genes (41), we questioned whether the stability of IncRNAs is different based on mRNA-like features, such as the 5' cap, 3' poly(A)-tail or sORF. For miRNA targeting analysis, the expression levels of the updated protein-coding mRNAs and IncRNAs were calculated using the RNA-seq by expectation-maximization, RSEM (42) (Supplementary Fig. S1; see Supplementary Material for more details). We compared the expression levels of IncRNAs to those of protein-coding mRNAs, revealing consistently higher expression of proteincoding mRNAs at all stages (Fig. 2A). We then compared the
A

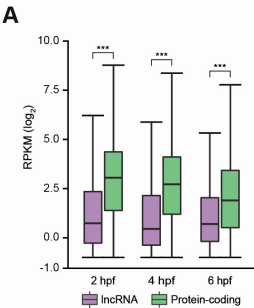

D

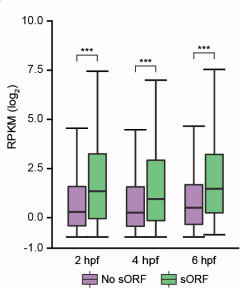

B
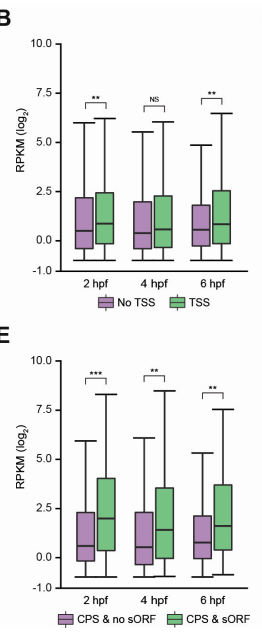
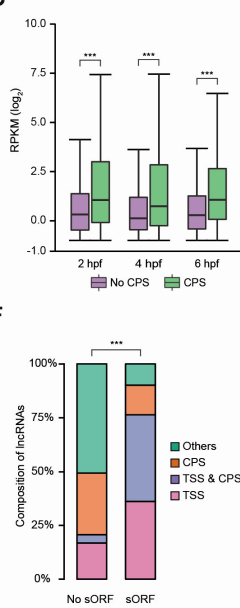

Fig. 2. Expression levels of IncRNAs with mRNA-like features at three developmental stages. (A-E) The expression levels of protein-coding mRNAs and IncRNAs are shown in box plots. The $y$-axis indicates the expression level, reads per kilobase of exons per million mapped reads (RPKM) in logarithm with base 2. The middle line in the boxes is the median level ${ }^{*} P \leq 0.05, * * P$ $\leq 0.01$, and $* * * P \leq 0.001$; NS, not significant; two-sided Kolmogorov Smirnov test) (A) Box plots showing expression levels for all protein-coding mRNA and IncRNA. (B-D) Box plots showing expression levels for subsets of IncRNAs with mRNA-like features. (B) The expression levels of IncRNAs with or without TSS are shown in box plots. (C) The expression levels of IncRNAs with or without CPS in box plots. (D) The expression levels of IncRNAs with or without SORF in box plots. (E) The expression levels of polyadenylated IncRNAs with or without sORF in the box plots. (F) The composition of IncRNAs with TSSs, CPSs, or both (*P $\leq 0.05, * * \mathrm{P} \leq 0.01$, and $* * * \mathrm{P} \leq$ 0.001; one-sided Fisher's exact test).
IncRNAs with no mRNA-like features to those with the features. While the expression levels of IncRNAs containing TSSs was similar to that of IncRNAs with no mRNA-like features at the 4 hours post-fertilization (hpf) stage (Fig. 2B; $P=0.210$ ), the IncRNAs containing CPSs were significantly more abundant and stable than those with no mRNA-like features at all stages (Fig. 2C; $\mathrm{P}=1.709 \times 10^{-9}$ for 2 hpf; $\mathrm{P}=$ $8.572 \times 10^{-10}$ for 4 hpf; $\mathrm{P}=1.215 \times 10^{-8}$ for $6 \mathrm{hpf}$ ). This observation agrees with previous in vitro results, showing that the existence of $3^{\prime}$ poly(A)-tail, the product of a cleavage and polyadenylation event (43), is an important determinant of RNA stability, where the poly(A)-binding protein (PABP) binds to the $3^{\prime}$ poly(A)-tail and stabilizes the RNA, whereas the RNA without a $3^{\prime}$ poly(A)-tail is quickly degraded (44). Interestingly, the IncRNAs with sORFs were more abundant and stable than those with no sORFs (Fig. 2D; $\mathrm{P}=9.302 \times 10^{-8}$ for $2 \mathrm{hpf}$; $\mathrm{P}=8.292 \times 10^{-6}$ for 4 hpf; $\mathrm{P}=6.951 \times 10^{-8}$ for $\left.6 \mathrm{hpf}\right)$. As the IncRNAs with CPSs were observed to be more stable and highly expressed, we suspected that the higher expression levels of IncRNAs with sORFs could be explained by the presence of CPS. Therefore, IncRNAs with CPSs and sORFs were compared to those with CPSs but no sORFs, revealing greater abundance and stability for IncRNAs with CPSs and sORFs (Fig. 2E). Taken together, these results are evidence in support of a $5^{\prime}$ cap, 3' poly (A)-tail and sORF being important determinants of IncRNA stability and expression levels. In fact, the IncRNAs with sORFs tended to have both TSS and CPS, indicating mRNA-like IncRNAs $\left(\mathrm{P}<2.200 \times 10^{-16}\right.$; onesided Fisher's exact test, Fig. 2F).

\section{The IncRNAs are affected by miRNAs at an early stage of development}

To investigate the impact of miRNA transfection on IncRNAs, we predicted canonical miRNA target sites, including $8 \mathrm{mer}$, $7 \mathrm{~m} 8,7 \mathrm{~A} 1$, and $6 \mathrm{mer}$ sites in the whole exons and $3^{\prime}$ UTRs of IncRNAs, and protein-coding mRNAs, respectively (Fig. 3A and Supplementary Fig. S3). As expected, the proportion of miR-132 and miR-155 site types was very similar between protein-coding mRNAs and IncRNAs (Fig. 3B). Analyses of the changes in RNA and RPF levels between mock- and miR-132/155-injected zebrafish embryos revealed that proteincoding mRNAs displayed translational repression in early zebrafish embryo stages (pre-MZT), at 2 and $4 \mathrm{hpf}$, but not at the $6 \mathrm{hpf}$ (Supplementary Fig. S4, bottom). Instead, the majority of the downregulation resulted from post-transcriptional repression at the $6 \mathrm{hpf}$ stage (Supplementary Fig. S4, top). However, IncRNAs showed significant repression in RNA levels at an earlier stage of development (2 hpf) (Fig. 3C, top). Although the change of RPF levels at the subsequent time (4 hpf) was significant (Supplementary Fig. S5), the change of the RPF level was mainly caused by the change of the RNA level, resulting in no change of translation efficiency (Fig. 3C, bottom), which is the change of RPF levels normalized by those of RNA. These results demonstrate that a substantial 
A
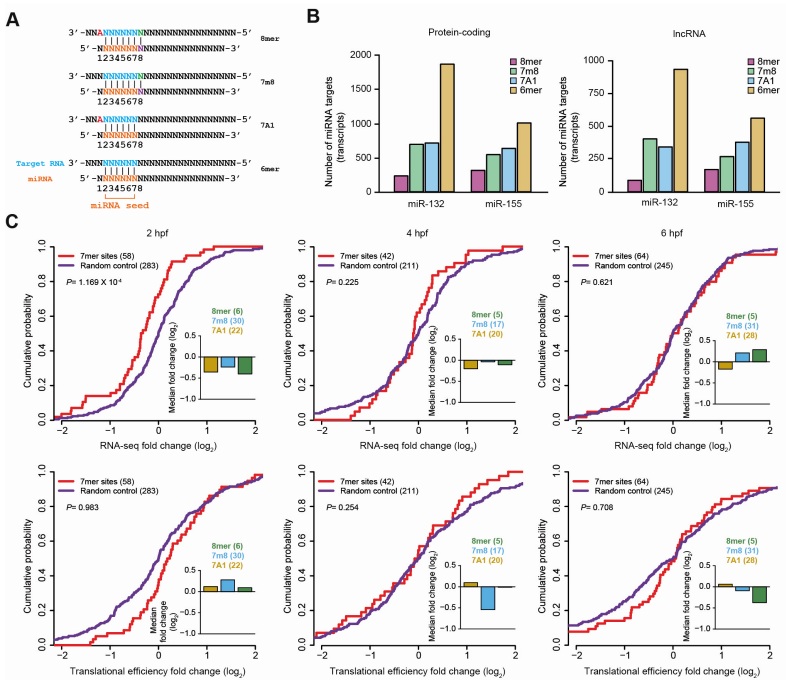

Fig. 3. miRNA targeting on IncRNAs. (A) Canonical miRNA target sites. (B) The number of protein-coding mRNAs (left) and IncRNAs (right) embedding miR-132 or miR-155 target sites. In case of multiple sites, only the site with a greater impact was considered as the site corresponding to the transcript. (C) The cumulative distribution functions (CDFs) show the changes of RNA expression (top) and translational efficiency (bottom) for IncRNAs with miRNA (red) and random target sites (purple) (see Supplementary Material for more details), at each developmental stage in the zebrafish embryos. The number of miRNA and random target sites are shown in parenthesis in top left corner. $P$ values were calculated using a one-tailed Wilcoxon rank-sum test and are shown at the top left corner. For each CDF, the median fold change $(\log 2)$ and the number of 7 mer sites of miR-132 and miR-155 (right), which are 8mer (green), 7m8 (sky blue) and 7A1 (dark orange), are depicted.

portion of IncRNA population might interact with miRNA, and are mostly affected in RNA levels at an early stage of development, compared to protein-coding genes.

\section{miRNA impacts mRNA-like IncRNAs}

We hypothesized that IncRNAs with mRNA-like features can be regulated by miRNA transfection in a different scale or manner, compared to other IncRNAs. To test this hypothesis, the subset of IncRNAs containing mRNA-like features was compared to those without these features, with respect to the response to miRNA transfection (Fig. 4A-C). Since the number of IncRNAs is much less than the number of protein-coding mRNAs, log2 fold changes of miR-132 and -155 targets, and of non-targets, were combined in the downstream analysis, to achieve predictive statistical-power for the IncRNA subgroups. The IncRNAs with TSS or CPS, which are the evidence of $5^{\prime}$ cap and $3^{\prime}$ poly(A)-tail respectively, exhibited significant repression by miRNA transfection in the RNA and RPF levels at 2 and $4 \mathrm{hpf}$ of zebrafish embryos, respectively, while IncRNA without TSS or CPS did not exhibit significant repression at any developmental stages of the zebrafish
A
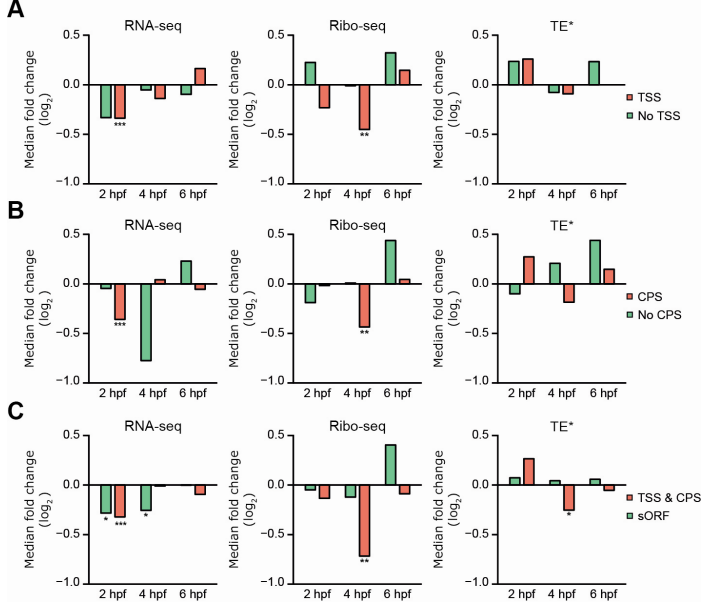

$\mathrm{TE}^{*}$

Fig. 4. miRNA-mediated repression of IncRNAs with $5^{\prime}$ cap and 3' poly(A)-tail. (A-C) Median fold changes of RNA (left), RPF (middle) levels and translational efficiency (asterisk, *TE in right) of IncRNAs containing canonical miR-132 and -155 target sites with or without mRNA-like features, such as TSS (A), CPS (B), sORF or both TSS and CPS (C). The $P$ values were calculated using one-tailed Wilcoxon rank sum test as in Fig. 3C, D. *P $\leq$ $0.05, * * P \leq 0.01$, and $* * * P \leq 0.001$.

embryos (Fig. 4A, B). For further assessment of IncRNA with mRNA-like features, IncRNAs with both TSS and CPS, and with SORF, were respectively analyzed in the same manner (Fig. 4C). IncRNAs with both TSS and CPS showed significant repression of RNA and RPF levels at $2 \mathrm{hpf}$ and $4 \mathrm{hpf}$ of zebrafish embryos, respectively. While those with sORF did not exhibit significant translational repression mediated by miRNA at any developmental stages, IncRNAs with TSS and CPS exhibited significant reduction in translational efficiency at $4 \mathrm{hpf}$ of zebrafish embryo (Fig. 4C). Taken together, these results support our hypothesis of miRNA-mediated repression on mRNA-like IncRNAs, as shown in exemplified IncRNAs containing miRNA target site with/without both TSS and CPS (Supplementary Fig. S6A, B). Note that ENSDART00000128177, a IncRNA classified as a processed transcript, supported by both TSS and CPS and predicted to contain miR-155 7m8 site, was downregulated in RNA and RPF levels at 2 hpf and $4 \mathrm{hpf}$ of miR-155 injected zebrafish embryos (Supplementary Fig. S6A), while ENSDART00000152905, a IncRNA classified as an antisense transcript not supported by TSS and CPS, and predicted to contain miR-155 8mer target site, was not (Supplementary Fig. S6B).

\section{IncRNAs with putative ORFs are affected by miRNA as mRNA-like IncRNAs}

In addition to IncRNAs with SORFs, the subsets of IncRNAs containing putative ORFs that possibly encode peptides longer than 100 amino acids, were separately analyzed (Supplementary Fig. S7). At all developmental stages in the zebrafish 
embryos, the expression levels of IncRNAs having putative ORFs were significantly higher than those lacking the evidence of any ORF (Supplementary Fig. S7A; $P=7.529 \times 10^{-13}$ for 2 hpf; $\mathrm{P}=2.913 \times 10^{-11}$ for 4 hpf; $\mathrm{P}=1.053 \times 10^{-4}$ for 6 hpf). Although the expression levels for IncRNAs containing putative SORFs or ORFs were comparable to each other throughout the developmental stages $(\mathrm{P}=0.062$ for $2 \mathrm{hpf} ; \mathrm{P}=$ 0.056 for $4 \mathrm{hpf}$; $\mathrm{P}=0.621$ for $6 \mathrm{hpf}$ ), their expression levels were much lower than that of protein-coding mRNAs at any stage (Supplementary Fig. S7A; $\mathrm{P}=8.742 \times 10^{-21}$ for $2 \mathrm{hpf}$; $\mathrm{P}=1.069 \times 10^{-22}$ for $4 \mathrm{hpf} ; \mathrm{P}=5.541 \times 10^{-5}$ for $6 \mathrm{hpf}$ ). Next, the IncRNAs with putative ORFs were analyzed for similar mode of miRNA targeting as that for IncRNAs with sORFs. Akin to the IncRNAs with sORF, those with the putative ORFs exhibited a similar pattern and degree of repression of RNA levels at $2 \mathrm{hpf}$, but no repression of RPF levels and translational efficiency at any other stage (Supplementary Fig. S7B).

\section{DISCUSSION}

We used the RNA-seq and Ribo-seq data from multiple developmental stages of zebrafish embryos (14), to study the interaction between miRNAs and IncRNAs with mRNA-like features. While there is an established pattern of MGS observed in protein-coding genes - early translational repression and late, predominant post-transcriptional repression $(10,13,14)$ - different pattern of MGS, the repression of RNA and RPF levels at $2 \mathrm{hpf}$ and $4 \mathrm{hpf}$ of zebrafish embryos, were observed in IncRNAs. A subsequent analysis of the IncRNA subsets containing TSS, CPS, or SORF signals revealed that the presence of $5^{\prime}$ cap and poly(A) tail might be required for efficient miRNA targeting and miRNA-mediated IncRNA repression at both the RNA and RPF levels. Post-transcriptional repression, observed on IncRNAs with both the TSS and CPS at an early stage of development $(\sim 2 \mathrm{hpf}$ of zebrafish embryo), compared to those on protein-coding mRNAs, could be explained by the relatively shorter half-lives of IncRNAs (45). What was interesting was significant translational repression of IncRNA with mRNA like features, 5' cap and 3' poly(A)-tail mediated by miRNA. This result coincides with the observation from the previous study that circular RNA ciRS-7, which lack both $5^{\prime}$ cap and $3^{\prime}$ poly(A)-tail structure, were not affected by miRNAs and worked as a functional miRNA decoy, while polyadenylated, linear construct of ciRS-7 exhibited miRNA-mediated repression (25). Therefore, we propose that the presence of a $5^{\prime}$ cap and $3^{\prime}$ poly(A)-tail in IncRNAs would be one of important determinants of miRNA-mediated repression in IncRNAs (Supplementary Fig. S8). As many IncRNAs contain the $5^{\prime}$ cap and $3^{\prime}$ poly(A)-tail (28), this miRNA-mediated repression of IncRNAs may represent a new regulatory regime for non-coding RNA networks. Moreover, as IncRNAs without those features were not significantly affected by miRNAs, it is possible that the subset of IncRNAs without $5^{\prime}$ cap and $3^{\prime}$ poly(A)-tail might work as functional miRNA decoys.

\section{MATERIALS AND METHODS}

Detailed information is provided in the Supplementary Material.

\section{ACKNOWLEDGEMENTS}

We thank all BIG Lab members for helpful discussions. This work was supported by the Basic Science Research Program through the National Research Foundation of Korea, and funded by the Ministry of Science, ICT \& Future Planning (NRF-2012M3A9D1054516) and supported by the Program for Agriculture Science \& Technology Development of the Rural Development Administration, Republic of Korea (Project No. PJ01045303).

\section{CONFLICTS OF INTEREST}

The authors have no conflicting financial interests.

\section{REFERENCES}

1. Bartel DP (2009) MicroRNAs: target recognition and regulatory functions. Cell 136, 215-233

2. Grimson A, Farh KK-H, Johnston WK, Garrett-Engele $P$, Lim LP and Bartel DP (2007) MicroRNA Targeting Specificity in Mammals: Determinants beyond Seed Pairing. Molecular Cell 27, 91-105

3. Baek D, Villén J, Shin C, Camargo FD, Gygi SP and Bartel DP (2008) The impact of microRNAs on protein output. Nature 455, 64-71

4. Shin C, Nam J-W, Farh KK-H, Chiang HR, Shkumatava A and Bartel DP (2010) Expanding the MicroRNA Targeting Code: Functional Sites with Centered Pairing. Mol Cell 38, 789-802

5. Garcia DM, Baek D, Shin C, Bell GW, Grimson A and Bartel DP (2011) Weak seed-pairing stability and high target-site abundance decrease the proficiency of Isy- 6 and other microRNAs. Nat Struct Mol Biol 18, 1139-1146

6. Agarwal V, Bell GW, Nam J-W and Bartel DP (2015) Predicting effective microRNA target sites in mammalian mRNAs. eLife 4, e05005

7. Bagga S, Bracht J, Hunter S et al (2005) Regulation by let-7 and lin-4 miRNAs Results in Target mRNA Degradation. Cell 122, 553-563

8. Lim LP, Lau NC, Garrett-Engele P et al (2005) Microarray analysis shows that some microRNAs downregulate large numbers of target mRNAs. Nature 433, 769-773

9. Lee RC, Feinbaum RL and Ambros V (1993) The C. elegans heterochronic gene lin-4 encodes small RNAs with antisense complementarity to lin-14. Cell 75, 843854

10. Guo H, Ingolia NT, Weissman JS and Bartel DP (2010) Mammalian microRNAs predominantly act to decrease 
target mRNA levels. Nature 466, 835-840

11. Eichhorn SW, Guo H, McGeary SE et al (2014) mRNA destabilization is the dominant effect of mammalian microRNAs by the time substantial repression ensues. Mol Cell 56, 104-115

12. Djuranovic S, Nahvi A and Green R (2012) miRNAmediated gene silencing by translational repression followed by mRNA deadenylation and decay. Science 336, 237-240

13. Bazzini AA, Lee MT and Giraldez AJ (2012) Ribosome profiling shows that miR-430 reduces translation before causing mRNA decay in zebrafish. Science 336, 233-237

14. Subtelny AO, Eichhorn SW, Chen GR, Sive $\mathrm{H}$ and Bartel DP (2014) Poly(A)-tail profiling reveals an embryonic switch in translational control. Nature 508, 66-71

15. Liu Q, Huang J, Zhou N et al (2013) LncRNA loc285194 is a p53-regulated tumor suppressor. Nucleic Acids Res 41, 4976-4987

16. Cesana M, Cacchiarelli D, Legnini I et al (2011) A Long Noncoding RNA Controls Muscle Differentiation by Functioning as a Competing Endogenous RNA. Cell 147, 358-369

17. Faghihi MA, Zhang M, Huang J et al (2010) Evidence for natural antisense transcript-mediated inhibition of microRNA function. Genome Biol 11, R56

18. Dey BK, Pfeifer K and Dutta A (2014) The H19 long noncoding RNA gives rise to microRNAs miR-675-3p and miR-675-5p to promote skeletal muscle differentiation and regeneration. Genes Dev 28, 491-501

19. Quinn JJ and Chang HY (2016) Unique features of long non-coding RNA biogenesis and function. Nat Rev Genet $17,47-62$

20. Denzler R, McGeary Sean E, Title Alexandra C, Agarwal V, Bartel David P and Stoffel M (2016) Impact of MicroRNA Levels, Target-Site Complementarity, and Cooperativity on Competing Endogenous RNA-Regulated Gene Expression. Mol Cell 64, 565-579

21. Denzler R, Agarwal V, Stefano J, Bartel David P and Stoffel M (2014) Assessing the ceRNA Hypothesis with Quantitative Measurements of miRNA and Target Abundance. Mol Cell 54, 766-776

22. Leucci E, Patella F, Waage J et al (2013) microRNA-9 targets the long non-coding RNA MALAT1 for degradation in the nucleus. Sci Rep 3, 2535

23. Poliseno L, Salmena L, Zhang J, Carver B, Haveman WJ and Pandolfi PP (2010) A coding-independent function of gene and pseudogene mRNAs regulates tumour biology. Nature 465, 1033-1038

24. Yoon JH, Abdelmohsen K, Srikantan S et al (2012) LincRNA-p21 suppresses target mRNA translation. Mol Cell 47, 648-655

25. Hansen TB, Jensen $\mathrm{TI}$, Clausen $\mathrm{BH}$ et al (2013) Natural RNA circles function as efficient microRNA sponges. Nature 495, 384-388

26. Franco-Zorrilla JM, Valli A, Todesco M et al (2007) Target mimicry provides a new mechanism for regulation of microRNA activity. Nat Genet 39, 1033-1037

27. Nam JW and Bartel DP (2012) Long non-coding RNAs in C. elegans. Genome Res 22, 2529-2540

28. Ulitsky I and Bartel David P (2013) lincRNAs: Genomics,
Evolution, and Mechanisms. Cell 154, 26-46

29. Bazzini AA, Johnstone TG, Christiano R et al (2014) Identification of small ORFs in vertebrates using ribosome footprinting and evolutionary conservation. EMBO J 33, 981-993

30. Calviello L, Mukherjee N, Wyler E et al (2016) Detecting actively translated open reading frames in ribosome profiling data. Nat Methods 13, 165-170

31. Ruiz-Orera J, Messeguer X, Subirana JA and Alba MM (2014) Long non-coding RNAs as a source of new peptides. Elife 3, e03523

32. Smith JE, Alvarez-Dominguez JR, Kline N et al (2014) Translation of small open reading frames within unannotated RNA transcripts in Saccharomyces cerevisiae. Cell Rep 7, 1858-1866

33. Andrews SJ and Rothnagel JA (2014) Emerging evidence for functional peptides encoded by short open reading frames. Nat Rev Genet 15, 193-204

34. Hezroni H, Koppstein D, Schwartz MG, Avrutin A, Bartel DP and Ulitsky I (2015) Principles of long noncoding RNA evolution derived from direct comparison of transcriptomes in 17 species. Cell Rep 11, 1110-1122

35. Pauli A, Valen E, Lin MF et al (2012) Systematic identification of long noncoding RNAs expressed during zebrafish embryogenesis. Genome Res 22, 577-591

36. Ulitsky I, Shkumatava A, Jan $\mathrm{CH}$, Sive $\mathrm{H}$ and Bartel DP (2011) Conserved function of lincRNAs in vertebrate embryonic development despite rapid sequence evolution. Cell 147, 1537-1550

37. Nepal C, Hadzhiev Y, Previti C et al (2013) Dynamic regulation of the transcription initiation landscape at single nucleotide resolution during vertebrate embryogenesis. Genome Res 23, 1938-1950

38. Jan CH, Friedman RC, Ruby JG and Bartel DP (2011) Formation, regulation and evolution of Caenorhabditis elegans 3'UTRs. Nature 469, 97-101

39. Kong L, Zhang Y, Ye ZQ et al (2007) CPC: assess the protein-coding potential of transcripts using sequence features and support vector machine. Nucleic Acids Res 35, W345-349

40. Derrien T, Johnson R, Bussotti G et al (2012) The GENCODE v7 catalog of human long noncoding RNAs: analysis of their gene structure, evolution, and expression. Genome Res 22, 1775-1789

41. Clark MB, Johnston RL, Inostroza-Ponta M et al (2012) Genome-wide analysis of long noncoding RNA stability. Genome Res 22, 885-898

42. Li B and Dewey CN (2011) RSEM: accurate transcript quantification from RNA-Seq data with or without a reference genome. BMC Bioinformatics 12, 323

43. Colgan DF and Manley JL (1997) Mechanism and regulation of mRNA polyadenylation. Genes Dev 11, 2755-2766

44. Wang Z and Kiledjian M (2000) The Poly(A)-Binding Protein and an mRNA Stability Protein Jointly Regulate an Endoribonuclease Activity. Mol Cell Biol 20, 6334-6341

45. Clark MB, Johnston RL, Inostroza-Ponta M et al (2012) Genome-wide analysis of long noncoding RNA stability. Genome Res 22, 885-898 